acrylic, locator, bar, cantilevered. The literature review is cross retrospective using quantitative and qualitative data.

Results and Conclusions: With the limitations of this study and the need for further evidence extracted as conclusions:

1. The need to assess patient personal factors: sex, power, approves arcade and expectations.

2. Distal portion of the posterior implants to the midline chin suffer greater stress load transmitted.

3. Option 4 implants placed in saltire offers better biomechanical performance.

\section{- Oral Presentation 42}

TITLE: Laser bioestimulation in the treatment of osteonecrosis associated with bisphosphonates

AUTHORS: Guenin Gutiérrez CV, Cabezas Mojón J, Esteban Adán G, Olivan Molina S, Loughney González A, Fernández Domínguez M.

Máster en Cirugía Bucal e Implantología. Universidad San Pablo CEU. Madrid

* doi:10.4317/medoral.17644036

http://dx.doi.org/10.4317/medoral.17644036

Introduction: exposure of necrotic maxillary or mandibular bone in patients treated with bisphosphonates is increasingly becoming a reason for dental consultation. Bisphosphonates act on osteoclasts inhibiting their chemotaxis, shortening their average life and inducing apoptosis. As a result, bone resorption stops.

Currently, we have a wide range of therapeutic options for the treatment of biphosphonate-induced osteonecrosis, being the laser therapy a simple, effective and well documented technique.

Aim: To review the laser therapy as a treatment for biphosphonate-induced osteonecrosis and to assess its effects.

Material and Methods: A bibliographic research was made in Pubmed database, using the following key words: bisphosphonates, osteonecrosis, osteonecrosis treatment, laser therapy. Among the reviewed articles, we include only the ones published in the last ten years.

Results: Many studies about bone biostimulation have shown the high effectiveness of $\mathrm{He}-\mathrm{Ne}$, Er:YAG and infrared diode lasers, among others. The authors agree that the application of a low intensity laser has a biostimulating effect on the reparative process, increasing the inorganic matrix of bone and the mitotic activity of osteoblasts leading to a significant improvement in clinical parameters.
Conclusions: Laser surgery is emerging as a valid therapeutic option for the osteonecrosis treatment and allows a minimally invasive approach in the early stages of the disease. More controlled clinical trials are required, in order to ensure a suitable treatment protocol for the patient.

\section{- Oral Presentation 43 \\ TITLE: Bisphosphonates \& Dental implant, success or failure? Bibliographic review, survey and cases presentation}

AUTHORS: Serfatty Castro G, Oliván Molina S, Martínez Albo D, Loughney González A, Fernández Domínguez M.

Máster en Cirugía Bucal e Implantología. Universidad San Pablo CEU. Madrid.

SOURCE: Med Oral Patol Oral Cir Bucal. 2016 December 15;21(Supplement1):S17.

* doi:10.4317/medoral.17644037

http://dx.doi.org/10.4317/medoral.17644037

Objective: Evaluate Implants treatment on Bisphophonate treated patients criteria, such as clinic experience, convergence and divergence between what is reported on and the specialist interviwed.

Material and Method: A bibliographic review was made, searching in odontological and medical data base such as: Pubmed, Medline and Scielo, using as key words: Dental Implants, Bisphophonates, Oseointegration, BRONM, only articles published after 2005 were included.

A Survey was conducted to specialist about: Dental Implants on Bisphophonates treated patiens and all the data was subject of statistic analysis.

We present 3 clinical cases of patients treated with Bisphophonates, who a CTX analysis were conduct to stablish the surgical risk of and implants treatment.

Findings: Most Articles consulted concludes that implant Osseointegration are not diminish by Bisphosphonate treatment, and no difference were found in the implant lost rate of this patients and the control patients.

The Survey indicates that $51 \%$ of the specialist place implants on Medicated patients, the $49 \%$ don't Agree on the use of that kind of treatment, there is a controversy referring tratments protocol and surgical risk determination, never the less the $71 \%$ agree about drug holydays as first option. The $22 \%$ of the participants don't Know CTX test, and the $51 \%$ consider that this test in not $100 \%$ Accurate.

Conclusion: Even though the specialized literature concludes that implant Osseointegration are not diminish by Bisphosphonate treatment, there is a real controversy 